\title{
An update on the safety and efficacy of decitabine in the treatment of myelodysplastic syndromes
}

This article was published in the following Dove Press journal:

OncoTargets and Therapy

24 February 2010

Number of times this article has been viewed

\author{
Jacqueline S Garcia' \\ Nitin Jain' \\ Lucy A Godley ${ }^{1,2}$ \\ 'Section of Hematology/Oncology, \\ Department of Medicine, \\ The University of Chicago, Chicago, \\ IL, USA; ${ }^{2}$ Cancer Research Center, \\ The University of Chicago, \\ Chicago, IL, USA
}

Correspondence: Lucy A Godley Section of Hematology/Oncology, Department of Medicine, The University of Chicago, 584I S. Maryland Avenue, MC 2I I5, Chicago, IL 60637, USA

$\mathrm{Tel}+\mathrm{I} 773-702-4 \mid 40$

Fax + I773-702-0963

Email lgodley@medicine.bsd.uchicago.edu

\begin{abstract}
Myelodysplastic syndromes (MDS) are clonal bone marrow malignancies characterized by peripheral cytopenias and dysplastic changes in the bone marrow with various clinical features. Patients with MDS, in particular those with intermediate-2 (Int-2) and high-risk disease, have a poor prognosis. The mainstay of treatment includes cytoxic chemotherapy and supportive care. Over the last decade, promising results from studies focusing on hypomethylating agents, such as decitabine (5-aza-deoxycytidine) and 5-azacitidine, have led to the expansion of the therapeutic arsenal for MDS. This review presents the current data available on the clinical efficacy and safety profile for decitabine as a treatment for MDS. Although not fully understood, decitabine's antitumor activity may involve its ability to induce hypomethylation and reactivation of genes responsible for cellular differentiation, stimulate an immune response, induce DNA damage/apoptotic response pathways, and/or augment stem cell renewal. Future studies that use epigenetic therapies that combine hypomethylating agents with histone deacetylase inhibitors (HDACi) and head-to-head comparison studies of decitabine and 5-azacitidine will provide valuable pre-clinical and clinical data, enhancing our understanding of these drugs.
\end{abstract}

Keywords: decitabine, 5-aza-deoxycytidine, 5-azacitidine, myelodysplastic syndromes

\section{Introduction}

Myelodysplastic syndromes (MDS) are classified as one of the five major categories of myeloid neoplasms according to the 2008 World Health Organization (WHO) classification system. ${ }^{1}$ MDS is a heterogeneous group of clonal stem cell disorders, which often feature a hypercellular bone marrow, peripheral cytopenias, and dysplasia in both peripheral blood and bone marrow. For the diagnosis of MDS, the minimal morphologic criteria include the identification of dysplasia in at least $10 \%$ of cells of any of the myeloid lineages in the peripheral blood/bone marrow after exclusion of acute myeloid leukemia (AML) and chronic myelomonocytic leukemia (CMML). ${ }^{2}$ MDS is chronic in most cases with the potential for worsened cytopenias over time due to bone marrow failure and progression to AML. More than 10,000 cases of MDS are diagnosed annually in the United States, with a median age of 71 years old. ${ }^{3}$

The International Prognostic Scoring System (IPSS) has been used as a clinical predictor of progression to AML. ${ }^{4}$ The IPSS uses three criteria to evaluate risk, including the percentage of blasts in the marrow, chromosomal abnormalities, and the number of cytopenias. Based on these criteria, a score of low-risk MDS, intermediate-1 (Int-1) risk, intermediate-2 (Int-2) risk, and high-risk MDS is assigned. Lower risk disease generally includes patients diagnosed with the WHO subtypes of refractory anemia, refractory anemia with ringed sideroblasts, refractory cytopenias with multilineage

submit your manuscript | www.dovepress.co 
dysplasia, MDS with del(5q), unclassifiable MDS, and patients assigned to IPSS low-risk and Int-1. Higher risk MDS includes patients with refractory anemia with excess blasts, and patients assigned to IPSS Int-2 and higher. Patients with MDS in general have a poor prognosis, with 3-year survival rates at a dismal $35 \% .^{3}$ In particular, those with higher-risk MDS have a survival rate of 0.4 to 1.2 years and a high risk for progression to AML. ${ }^{5}$

Currently, there is no consensus for treating patients with higher-risk MDS. Prior to the widespread use of the hypomethylating agents, decitabine (5-aza- 2 '-deoxycytidine) and 5-azacitidine, cytotoxic therapy or supportive care were the traditional mainstays of therapy. Supportive care offered to patients with MDS includes blood transfusions, administration of hematopoietic growth factors, and antibiotics for prophylaxis or treatment of opportunistic infections. Unfortunately, patients receiving these therapies often die of complications of cytopenias, progressive disease, and/or iron overload. Hematopoietic cell transplantation (HCT) is the only potentially curative therapy available. However, because the vast majority of MDS patients are over the age of 65 years with co-morbidities, intensive treatment options, such as induction chemotherapy and allogeneic HCT, are not feasible for many patients.

In the last two decades, the use of epigenetic therapy, such as decitabine, has gained popularity, given its favorable side effect profile and its potential to improve survival. ${ }^{6,7}$ Decitabine was approved by the US Food and Drug Administration (FDA) in 2006 for the treatment of de novo and secondary MDS (treatment-related MDS, t-MDS). ${ }^{8}$ Here we present a review of the clinical efficacy and safety of decitabine in the treatment of MDS with an explicit focus on the clinical trials published since Kantarjian et al's multiinstitutional, Phase III randomized study comparing clinical outcomes after treatment with decitabine versus best supportive care for patients diagnosed with MDS (Table 1). ${ }^{9}$

\section{Pharmacology of decitabine}

Decitabine contains deoxyribose as its sugar base and is incorporated into DNA (Figure 1A). The drug is considered to be chemically unstable when in basic or acidic solutions, but at neutral $\mathrm{pH}$, it has a half-life of 7 days at $4^{\circ} \mathrm{C}, 96$ hours at $20^{\circ} \mathrm{C}$, and 21 hours at $37^{\circ} \mathrm{C} \cdot .^{10,11}$ Once inside the cell, decitabine needs to be activated to 5 -aza- 2 -deoxycytidine$5^{\prime}$-triphosphate. The rate-limiting step in this process is the conversion of decitabine to 5-aza- $2^{\prime}$-deoxycytidine$5^{\prime}$-monophosphate, a reaction catalyzed by deoxycytidine kinase. Subsequent phosphorylation events yield the triphosphate form, which can be incorporated at positions in DNA that normally contain cytidine.

5-azacitidine is chemically related to decitabine. It contains a ribose sugar ring, and as such, is incorporated largely into RNA and at a lower frequency into DNA. Once incorporated into DNA, its mode of action is thought to be similar to decitabine.

Only cytosine (or 5-azacitidine, azaC) residues followed by guanines are substrates for DNA methylation in somatic cells. Once the DNA methyltransferase enzymes (DNMT) recognize an azaC-G dinucleotide, they become trapped covalently to the cytosine residue, since the enzymes are unable to complete the enzymatic reaction due to the nitrogen at the 5-position (Figure 1B). Once covalently attached to the DNA, the mechanisms by which azaC mediates hypomethylation are not yet entirely clear. A widely held model postulates that trapped DNMT enzymes result in enzyme degradation, lower DNMT levels, and ultimately, hypomethylation.

The clinical activity of decitabine and its demethylating activity are not fully understood. Hypotheses regarding how hypomethylation and/or changes to chromatin structure result in clinical remissions include: reversal of cancer-associated hypermethylation events, induction of cellular differentiation, effects on stem cell populations, changes in the rate of apoptosis, induction of an immune response, and/or induction of DNA damage response pathways. ${ }^{12}$ Therefore, the hypomethylating drugs lower DNA methylation levels and alter chromatin structure, and much research still remains to be done to elucidate the molecular mechanism underlying clinical remissions.

DNA methylation and histone modification are both examples of potentially reversible epigenetic changes that can alter gene expression patterns. One mechanism of epigenetic gene silencing that is not fully understood includes hypermethylation of DNA sequences called $\mathrm{CpG}$ islands, which contain a high concentration of $\mathrm{CpG}$ dinucleotides and often overlap gene promoters. Methylated $\mathrm{CpG}$ islands bind specific proteins to recruit transcriptional corepressors such as histone deacetylases (HDACs). Removal of the acetyl groups by HDACs from the lysine tails of the histones leads to gene silencing. Thus, both DNA methylation and histone deacetylation are associated with gene silencing, providing a rationale for the combining a hypomethylating agent with a histone deacetylase inhibitor in order to induce clinical remissions, by re-activating gene expression, at least in theory.

Several clinical trials using decitabine, or more extensively 5-azacitidine, have incorporated correlative studies to 


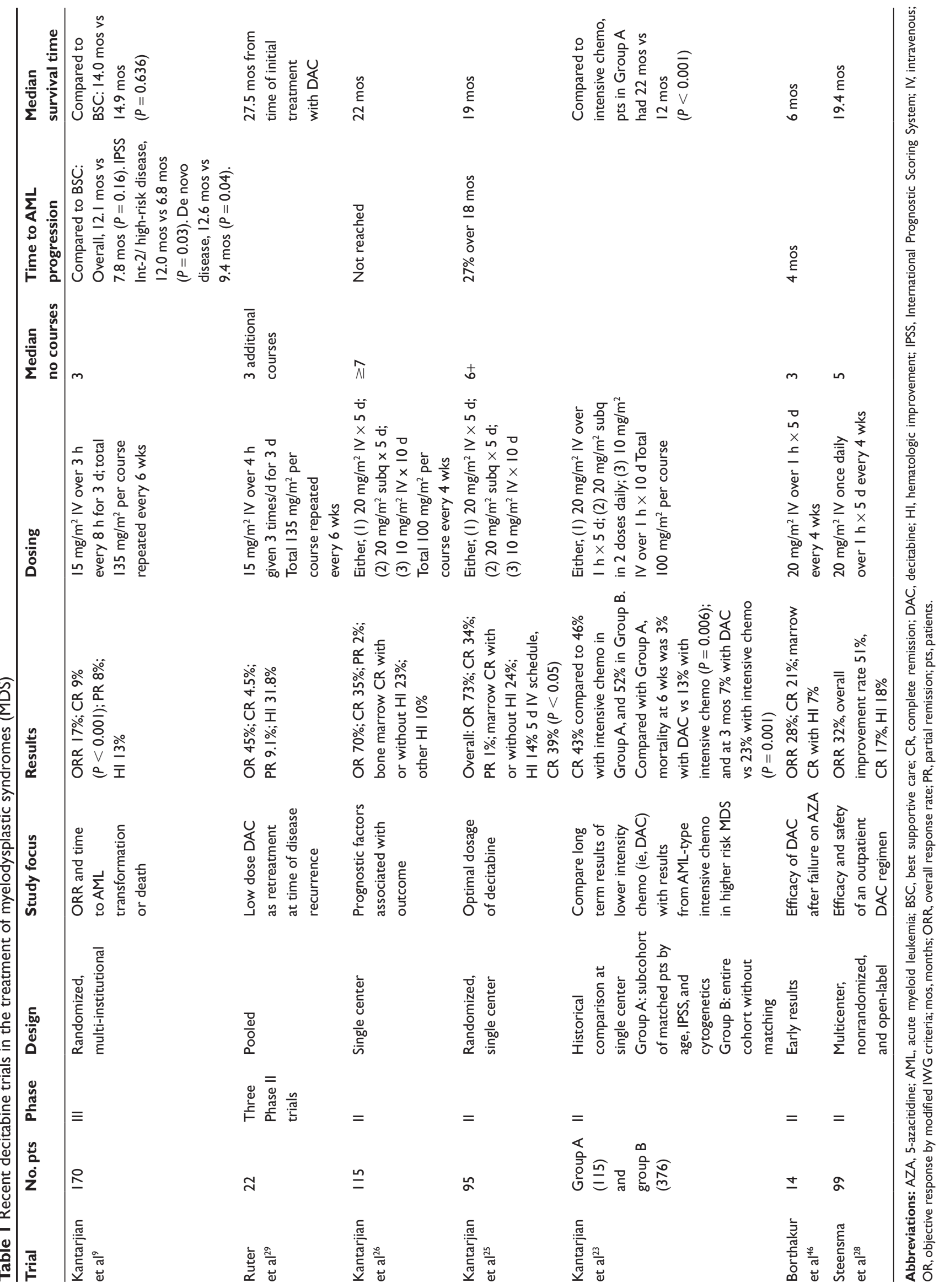


A

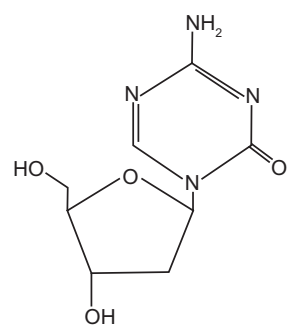

B DNMT

$$
\text { covalently }
$$$$
\begin{aligned}
& \text { bonds to } \\
& \text { cytosine }
\end{aligned}
$$

residue

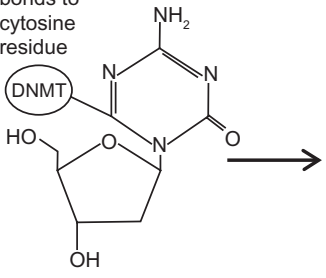

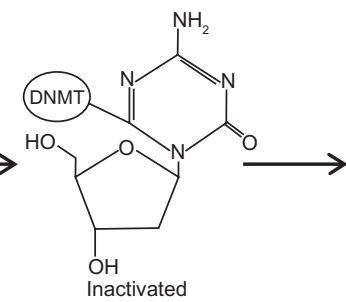

Figure I Structure and trapping mechanism of decitabine.A) The chemical structure of decitabine (4-amino-I-(2-deoxy- $\beta$-D-erythro-pentofuranosyl)-I,3,5-triazin-2 $(\mathrm{IH})$-one $\mathrm{C}_{8} \mathrm{H}_{12} \mathrm{~N}_{4} \mathrm{O}_{4}$ ). B) The action of the DNMTs is inhibited by decitabine-incorporated DNA. Covalently trapped DNMTs are degraded, leading to depletion of cellular DNMTs.

try to assess the biochemical effects of these drugs in patients. In two Phase I studies that tested a hypomethylating agent in combination with a histone deacetylase inhibitor (HDACi), although treatment with a hypomethylating agent resulted in increased levels of acetylated histones, there was no further increase after treatment with the HDACi. ${ }^{13,14}$ Therefore, there is little evidence currently that the hypomethylating agents are synergistic with HDACis as theorized. In another Phase I trial of 5-azacitidine combined with entinostat, another HDACi, bone marrow cells were tested for DNA methylation across 14,000 promoter regions using a microarray based assay, and DNA hypomethylation was present by day 15 and persisted to day 29, with DNA hypomethylation occurring across all chromosomal regions, often in areas containing SINE/Alu repetitive elements. ${ }^{15}$

\section{Clinical efficacy as first-line therapy for MDS}

In the past, the heterogeneic clinical/pathologic features and cytogenetic abnormalities of MDS presented a challenge to consistent outcome evaluation after treatment. To address this problem, an International Working Group (IWG) of investigators established response criteria in myelodysplasia in $2000 .{ }^{16}$ The IWG response criteria have been adopted widely, and hence, provide clinicians with a useful standardized tool to measure the efficacy of decitabine, for instance, in the treatment of MDS. Complete remission (CR) in the bone marrow is defined as fewer than 5\% blasts in the bone marrow without evidence of dysplasia, and in the peripheral blood is defined as: neutrophils of $1500 / \mathrm{mm}^{3}$ or more, a hemoglobin greater than $11 \mathrm{~g} / \mathrm{dL}$, platelets of $100,000 / \mathrm{mm}^{3}$ or more, absence of blasts, and no dysplasia for at least 8 weeks. To achieve partial remission (PR), CR criteria must be met if abnormal prior to therapy, except that bone marrow blasts must decrease by $50 \%$ or more compared to pretreatment levels for at least 8 weeks. Cytogenetic response is divided into two categories: major, for responses in which there is no detectable cytogenetic abnormality, and minor, for responses in which there is $50 \%$ or more reduction in the number of abnormal metaphases. Hematologic improvement (HI) of at least 8 weeks is defined by major and minor categories. Major HI includes a $100 \%$ increase in the neutrophil count, or an increase of at least $500 / \mu \mathrm{L}$ if the baseline neutrophil count was less than $1500 / \mu \mathrm{L}$; transfusion independence, or an increase of at least $2 \mathrm{~g} / \mathrm{dL}$ in hemoglobin level; and for platelets, it is defined as transfusion independence, or an increase of $30,000 / \mu \mathrm{L}$, if the baseline platelet count was less than $100,000 / \mu \mathrm{L}$. Modifications of the IWG response criteria in 2006 included, among others: consolidating major and minor $\mathrm{HI}$ into clinically relevant $\mathrm{HI}$, changing duration of CR or PR to a minimum of 4 weeks instead of 8 weeks, permitting persistent dysplasia in $\mathrm{CR}$, including marrow $\mathrm{CR}$ (mCR) without recovery of counts as a new category, and defining of AML as at least $20 \%$ or more blasts by WHO classification. ${ }^{17}$ These criteria have been adopted in the studies described below, and they allow for comparisons across the clinical trials.

Decitabine was initially studied as a cytotoxic agent at doses of $1500-2500 \mathrm{mg} / \mathrm{m}^{2}$ per course. This led to delayed and prolonged myelosuppression despite demonstrating activity in leukemias. ${ }^{18-20}$ In contrast to high doses where decitabine has cytotoxic effects, selective DNA demethylating activity was seen at much lower doses, around 100 to $150 \mathrm{mg} / \mathrm{m}^{2}$ per course. ${ }^{8}$ Zagonel et al studied two low-dose schedules of decitabine (45 to $50 \mathrm{mg} / \mathrm{m}^{2} /$ day for 3 days) in 10 patients with MDS and 4 of these patients achieved CR. ${ }^{21}$ This and other studies exploring low-dose decitabine led to a larger Phase II trial by Wijermans and colleagues ${ }^{22}$ using a different dosing schedule of $15 \mathrm{mg} / \mathrm{m}^{2}$ infused over a 4-hour period every 8 hours (total of $45 \mathrm{mg} / \mathrm{m}^{2} /$ day) for 3 consecutive days every 6 weeks, in which results demonstrated an overall response rate $(\mathrm{ORR}=\mathrm{CR}+\mathrm{PR})$ of $49 \%$ with a $\mathrm{CR}$ 
of $20 \%$ and a PR of $4 \%$. A pooled analysis of the Phase II studies from Europe has also been published. ${ }^{6}$ In this analysis, 177 patients received decitabine 40 to $50 \mathrm{mg} / \mathrm{m}^{2} /$ day for 3 days every 6 weeks leading to an ORR of $49 \%$ with a CR of $24 \%$, a PR of $10 \%$, and an HI of $14 \%$.

These encouraging results led to the landmark multiinstitutional Phase III trial in the United States where 170 patients with de novo or t-MDS were randomized to receive decitabine plus supportive care versus supportive care alone. ${ }^{9}$ Decitabine was administered as a scheduled dose of $15 \mathrm{mg} / \mathrm{m}^{2}$ every 8 hours intravenously daily for 3 days every 6 weeks. The primary endpoints of the study were ORR and time to AML transformation or death. Best supportive care included blood transfusions for patients with hemoglobin $<8 \mathrm{~g} / \mathrm{dL}$, platelet transfusions for platelets $<7.5 \times 10^{9} / \mathrm{L}$, and administering hematopoietic colony-stimulating factors per study guidelines. Results of an intention to treat analysis of the total study population demonstrated that decitabine had a significantly better ORR as compared to patients on the supportive care arm $(17 \%$ versus $0 \%, P<0.001)$, with a CR of $9 \%$ and a PR of $8 \%$. Further, an HI was seen in $13 \%$ of patients on the decitabine arm compared to $7 \%$ of patients treated with supportive care $(P<0.001)$. Further, although not statistically significant, patients treated with decitabine had a delay to AML transformation or death of 4.3 months $(P=0.16)$. In the subgroup analysis, patients who were treatment-naïve, had an Int-2/high-risk IPSS risk score, or had de novo MDS, benefitted the most. The study design limited the number of courses of decitabine since patients who maintained a CR for 2 cycles were removed from the therapy arm. Due to myelosuppressive side effects, 18 patients had their treatment interrupted. A median of 3 courses of decitabine was administered. Of the group receiving decitabine, 52\% received greater or at least 3 courses and 26\% received greater than 6 courses. Treatment with decitabine led to a trend of RBC transfusion-independence when compared to best supportive care. Statistically significant sustainable improvements in global health status, fatigue, and dyspnea were detected in patients treated with decitabine. ${ }^{9}$ Based on this pivotal study, the FDA approved decitabine in May of 2006 for patients with for MDS (Int-1 or higher IPSS class) at the dose of $15 \mathrm{mg} / \mathrm{m}^{2}$ every 8 hours intravenously daily for 3 days every 6 weeks.

Long-term results of decitabine have not been compared to chemotherapy in higher-risk MDS. Specifically, the survival advantage with treating patients diagnosed with higher-risk MDS with decitabine is unknown. Kantarjian et al's subsequent single-center study comparing the efficacy and toxicity of decitabine to a historical control group treated with intensive chemotherapy in higher-risk MDS suggested a survival advantage associated with decitabine and prompted attention to the need for prospective analysis. ${ }^{23}$ This study analyzed the decitabine treatment arm to two historic intensive chemotherapy groups, one that matched each patient receiving decitabine to a patient from the intensive chemotherapy group with similar age, chromosomal abnormalities, and IPSS risk group prior to treatment, and the other study group included the entire cohort of 376 identified historic patients treated with intensive chemotherapy. Results demonstrated a statistically significant overall survival advantage of 22 months for those in the decitabine arm, compared to 12 months in the first intensive chemotherapy group with matched characteristics, and a 2-year survival advantage of $47 \%$ versus $24 \%(P<0.001)$. In particular, patients greater than 60 years of age had a 2-year survival rate of $40 \%$ versus $20 \%$ with decitabine $(P=0.001)$. In comparison to the second group of patients who received intensive chemotherapy, patients treated with decitabine demonstrated an overall survival advantage at 2 years with $47 \%$ versus 21\% $(P<0.0001)$. Patients with poor prognostic factors of chromosome 5 or 7 abnormalities, thrombocytopenia, and older age did not have a statistically significant survival advantage with decitabine $(P=0.06)$. Despite the suggested survival advantage data from this historical comparison with decitabine, the CR rate was lower in this treatment arm.

Survival was again assessed as an endpoint in the follow-up randomized, multi-centered Phase III study of the EORTC Leukemia and German MDS Study Groups comparing overall survival with low dose decitabine versus supportive care in patients over 60 years old diagnosed with primary or secondary MDS or CMML. ${ }^{24}$ Those on the decitabine arm were given a treatment schedule of $15 \mathrm{mg} / \mathrm{m}^{2}$ intravenously over 4 hours every 8 hours for the first 3 consecutive days of every 6-week cycle, for a maximum of 8 cycles. Poor risk cytogenetic abnormalities were detected in $46 \%$ of the patients. Patients were given a median of 4 cycles of decitabine, with $40 \%$ of them getting no more than 2 cycles. Results of this trial demonstrated an ORR of $34 \%$. There was no significant difference in the decitabine versus supportive care arm regarding time to AML progression or death. Overall survival was lower and was not found to be statistically significant, likely due to the shorter treatment duration or to the subsequent therapy given, such as transplant or induction chemotherapy, to patients with disease progression.

The response rate from these decitabine studies has been modest, and by the time of these studies, the optimal dose 
of decitabine was not yet determined. With the intent of achieving maximal efficacy and minimal toxicity, a singlecenter randomized Phase II study was performed in which 95 patients with either higher-risk MDS or CMML received 1 of 3 schedules of low-dose decitabine. ${ }^{25}$ Randomized schedules included $20 \mathrm{mg} / \mathrm{m}^{2}$ intravenously daily for 5 days, $20 \mathrm{mg} / \mathrm{m}^{2}$ subcutaneously daily for 5 days, and $10 \mathrm{mg} / \mathrm{m}^{2}$ intravenously daily for 10 days. Results demonstrated that $34 \%$ of patients in the study achieved CR, and $73 \%$ had an objective response according to the modified IWG criteria. In an attempt to correlate clinical activity of decitabine to its epigenetic activity in vivo, the authors studied global LINE1 methylation patterns and the level of $p 15$ activation expressed in treated patients. Data from this study suggested that decitabine had in vivo hypomethylating activity. The superior clinical arm was associated with the most rapid and profound induction of hypomethylation as well as induction of $p 15^{I N K 4 B}$. The authors concluded that a 5-day course of intravenous therapy at a dose of $20 \mathrm{mg} / \mathrm{m}^{2}$ daily was superior at inducing hypomethylation at day 5 and at activating $p 15$ expression at days 12 or 28 after therapy. ${ }^{25}$

In an update of these data, the authors reported a median survival of 22 months, with an estimated 2-year survival rate of $47 \% .{ }^{26}$ Given the results of the optimal dosing schedule, an adaptive randomization strategy was used to select the regimen of $20 \mathrm{mg} / \mathrm{m}^{2}$ intravenously daily for 5 days, due to the associated higher CR rate in this and previous studies. Interestingly, patients required a median number of 7 courses to achieve $\mathrm{CR}$. The $\mathrm{CR}$ rate was $35 \%$ with decitabine, and the ORR was $70 \%$. In addition to these findings, the authors identified prognostic factors associated with response and survival. As determined by multivariate analysis, poor prognostic factors identified for achieving $\mathrm{CR}$ as determined by IWG criteria included MDS versus CMML, longer history of MDS, and previous MDS therapy. Poor prognostic factors for survival included chromosome 5 and/or 7 abnormalities, older age, and previous MDS therapy. Despite the poor prognosis associated with chromosome 5 and 7 abnormalities, Ravandi et al demonstrated improved overall survival with hypomethylating agents when compared to intensive chemotherapy for treatment of AML and high-risk MDS with those cytogenetic features. ${ }^{27}$ Results demonstrated that $41 \%$ of patients in the hypomethylating arm achieved CR versus $35 \%$ of patients in the chemotherapy group $(P=0.395)$. Specifically, 56 patients were treated with decitabine alone and 23 achieved CR. Patients treated with a hypomethylating agent had a survival advantage when compared to those who received cytotoxic chemotherapy $(P=0.019)$. Hence, patients diagnosed with difficult to treat MDS, such as those with the poor prognostic factors, have been shown to benefit from decitabine when compared to intensive chemotherapy.

More recently, robust data collected from the Alternative Dosing for Outpatient Treatment (ADOPT) trial demonstrated clinical benefit of decitabine to patients with MDS. This multicenter, nonrandomized, and open-label trial tested the efficacy and safety of an outpatient regimen of $20 \mathrm{mg} / \mathrm{m}^{2}$ of decitabine infused intravenously over 1 hour daily for 5 consecutive days every 4 weeks using ORR as the primary endpoint. ${ }^{28}$ Patients with a median age of 72 years were enrolled. Using IPSS risk scoring, study investigators identified 23\% high, 23\% Int-2, 53\% Int-1, and 1\% lowrisk persons among the group. The study design excluded dose escalations or reductions. Supportive care including transfusions was permitted and used according to physician discretion. For the 99 patients enrolled at 28 North American sites, the ORR was 32\% (95\% CI, 23\% to 43\%), and 18 of 99 patients had HI (95\% CI, 40\% to 61\%). The median time to initial clinical improvement was detected after the first 2 cycles, and the median duration of improvement was 10 months. This study showed that $24 \%$ of patients required more than 5 cycles to achieve best response, further advocating the need for repeated drug exposure over several cycles to achieve therapeutic benefit. Among the various IPSS risk groups in this study, clinical benefit was shown in $50 \%$ of those known to be intermediate-1, $61 \%$ of those identified as intermediate- 2 , and $43 \%$ for high-risk patients. There was an impressive rate of $73 \%$ improvement among CMML patients, defined as $\mathrm{CR}+\mathrm{mCR}+\mathrm{PR}+\mathrm{HI}$. Further, among the 33 patients with abnormal baseline cytogenetic analyses, there was a $52 \%$ cytogenetic response rate. Data collected from this study demonstrated a median survival of 19.4 months by the closure of the study. The most common reported side effect was cytopenias. Studies prior to the ADOPT trial committed patients to frequent hospitalizations. Encouraging results from this study demonstrated that outpatient decitabine is comparable to the inpatient regimen tested in previous Phase III studies, ${ }^{9,24}$ and it affirmed the efficacy of the scheduling dose of $20 \mathrm{mg} / \mathrm{m}^{2}$ intravenously for 5 days every 4 weeks in Kantarjian's single-center study ${ }^{23}$ discussed above. This is now considered a standard outpatient regimen for decitabine.

In summary, the current trials of decitabine have shown modest clinical efficacy (ORR $17 \%$ to $32 \%$ ) in patients with higher-risk MDS. Optimizing the dosing schedule of decitabine to maximize its hypomethylating effect includes using it at a low dose, at high dose intensity, and 
in multiple cycles. Data demonstrating a survival advantage with the use of decitabine when compared to best supportive care have been analyzed only retrospectively.

\section{Clinical efficacy in recurrent disease}

For patients diagnosed with recurrent disease, decitabine remains a viable option given its low nonhematologic toxicity profile, which allows for repeated courses. The studies above demonstrated that at least 3 courses of decitabine were needed to achieve efficacy. In an earlier study combining 3 Phase II trials, the outcome of re-treatment with decitabine was studied in 22 high-risk MDS patients with low-dose decitabine at $15 \mathrm{mg} / \mathrm{m}^{2}$ over 4 hours given 3 times a day on 3 consecutive days for up to 6 to 8 courses at the time of disease recurrence. ${ }^{29}$ Sixty percent of these patients achieved a clinical response (CR, PR, or $\mathrm{HI})$ after receiving a median of 6 courses of decitabine. Of the 65 patients who responded well to decitabine, 22 patients with recurrent disease demonstrated an inferior response upon subsequent exposure. The remaining 43 patients with recurrent disease were treated with induction chemotherapy or best supportive care, with or without low-dose chemotherapy. In 10 of the 22 patients with recurrent disease who were given re-treatment with decitabine, 7 patients achieved a HI, 2 patients achieved a PR, and 1 patient achieved a second $\mathrm{CR}$. The duration of this second response was 4 months, approximately 2.5 times shorter in duration than on the first response. Of the remaining 12 patients with recurrent disease who were offered decitabine again, resistance was evident in 4 patients. Altogether, $45 \%$ of patients receiving decitabine again were still treatment sensitive. Although the focus of this study was re-treatment effects and the potential of decitabine as maintenance therapy, the data strongly suggest prolonging initial therapy. ${ }^{29}$

\section{Outcomes in lower-risk MDS and CMML}

Management of patients with lower risk MDS relies primarily on supportive care and hematopoietic growth factors, particularly erythropoiesis-stimulating agents. Patients with $5 q-$ Syndrome are often treated with lenalidomide, which is considered standard of care. ${ }^{30}$ Decitabine is considered for patients with symptomatic anemia who fail to respond to agents like darbepoeitin or granulocyte colony-stimulating factor. Limited data are available demonstrating the clinical efficacy of decitabine in these diseases. However, given the risk of iron overload and its complications related to hepatic, cardiac, and endocrine function, as well as the potential need to treat with deferoxamine, decitabine represents a potential alternative and may obviate the degree of supportive care. A Phase II trial sponsored by the CALGB focused on the clinical efficacy of subcutaneous decitabine to adults with low or intermediate-1 risk MDS is ongoing (Clinicaltrials.gov Identifier NCT00619099), and may provide further insight on its efficacy in lower risk MDS.

CMML is an uncommon clonal disorder of the bone marrow that has been classified as a myelodysplastic/myeloproliferative (MDS/MPS) according to WHO classification given its heterogeneous clinical, hematological and morphologic features. $^{2}$ The median survival in CMML is 18 to 20 months from diagnosis. ${ }^{31}$ Subset analysis on patients diagnosed with CMML from existing decitabine trials has offered invaluable insight on the management of a disease that is difficult to treat as there are few studies to date that study CMML alone. The experience with decitabine in CMML was demonstrated in three open-label and single-arm multicenter Phase II studies (PCH 91-1, ${ }^{32} \mathrm{PCH} 95-11,{ }^{22} \mathrm{PCH}$ 97-196) and a multicenter Phase III study (D-0007 ${ }^{9}$ ) retrospectively reviewed by Wijermans et al. ${ }^{33}$ Overall, results from those 4 studies demonstrated an ORR of $26 \%(10 \%$ $\mathrm{CR}+16 \%$ PR), a HI of $19 \%$, a median of 15 -month survival from initial decitabine treatment ( $95 \% \mathrm{CI}, 8$ to 22 months), and a 2 -year survival of $25 \%$. In the trial conducted by Aribi et al to evaluate the activity of decitabine in patients diagnosed with CMML, 19 patients with CMML were given 1 of 3 schedules of decitabine with total dose per course of $100 \mathrm{mg} / \mathrm{m}^{2} .^{34}$ The study design dictated repeated courses every 4 weeks for a minimum of 3 courses without dose escalations. Results demonstrated an ORR of $68 \%$, a CR of $58 \%$, a HI of $11 \%$, and a 2 -year survival rate of $48 \%$. Patients were exposed to a median of 9 courses of decitabine. Larger randomized trials featuring patients with CMML are needed to further establish the potential of decitabine for this difficult to treat disease.

\section{Future of decitabine with HCT}

Most patients with intermediate and high-risk MDS are not candidates for allogeneic HCT because of their age and co-morbidities. It is not clear whether or not induction chemotherapy should be used routinely before undergoing HCT. The current 3-year survival rate of HCT in patients with less advanced MDS is $65 \%$ to $80 \%$ with human leukocyte antigen-identical related and unrelated donors, and 35\%-50\% in those with advanced MDS (defined as greater or equal to $5 \%$ marrow blasts) from related donors versus $25 \%$ to $40 \%$ 
from unrelated donors. ${ }^{35}$ Failure to benefit from HCT is likely due to treatment-related mortality or to relapse. Decreasing the toxicity or dose reduction of conditioning regimens has reduced the treatment-related mortality but has had a minimal effect on the risk of relapse.

Alternative regimens that can potentially alter the natural course of MDS include hypomethylating agents, histonedeacetylase inhibitors, or lenalidomide. ${ }^{36}$ These agents have been used as mono-versus combination therapy to improve the pre-transplant remission status prior to HCT or have been given post-transplant to prevent relapse in the form of maintenance or consolidation therapy. ${ }^{37-40}$ In a study evaluating the outcomes of 17 patients with MDS who underwent an allogeneic HCT after previous exposure to decitabine, results did not demonstrate increased toxicity with decitabine. ${ }^{41}$ Further, 100 days after transplant, 13 of 17 patients who underwent an allogeneic HCT were in CR, and 1 year later, 11 of these 13 patients were alive with 8 patients in CR and 3 patients with progressive disease. Several ongoing and planned clinical trials will also evaluate the role of decitabine as maintenance therapy after HCT.

\section{Safety and tolerability}

In the above described Phase II and Phase III trials, decitabine was generally safe and well tolerated. At its therapeutic dose, decitabine has favorable and manageable side effects, making it amenable to the elderly patient population in the treatment of MDS. Table 2 summarizes the toxicities seen in the studies discussed above using the NCI Common Toxicity Criteria for Adverse Events. The most common grade 1-2 adverse events reported included bleeding, fatigue, and nausea. Of the grade 3-4 side effects, the most frequent were neutropenia and thrombocytopenia. Given the myelosuppressive effects, it is recommended that patients continue antifungal and antibiotic prophylaxis while on decitabine. ${ }^{42}$ Cardiovascular and gastrointestinal abnormalities, in particular, are uncommon. The poorly tolerated cytotoxic side effects of mucositis, hair loss, diarrhea, and renal failure are uncommon with the use

Table 2 Reported adverse effects of decitabine

\begin{tabular}{|c|c|c|c|}
\hline Adverse event & Grade $1 \%-2 \%$ affected & Grade $3 \%-4 \%$ affected & Reference \\
\hline Neutropenia & 1 & $31,86 *$ & 9,28 \\
\hline Thrombocytopenia & 2 & $18,85^{*}$ & 9,28 \\
\hline Anemia & 5 & $12^{*}$ & 9,28 \\
\hline Bleeding & 46 & 7 & 25 \\
\hline $\begin{array}{l}\text { Febrile neutropenia, } \\
\text { fever of unknown origin }\end{array}$ & 3 & $6,14,20,23^{*}, 33$ & $6,9,25,28,34,46$ \\
\hline Leukopenia & & $22^{*}$ & 9 \\
\hline Pyrexia & & $6^{*}$ & 9 \\
\hline Liver dysfunction & $2,10,11$ & $\mathrm{I}, \mathrm{I}, 4,6^{*}$ & $6,9,25,26,34$ \\
\hline Pneumonia & I & $2,11,15^{*}, 20$ & $6,9,25,28,34$ \\
\hline Nausea & $2,17,26$ & $0, I^{*}, 7$ & $6,9,25,26,28,34$ \\
\hline Pyrexia & 17 & 0 & 28 \\
\hline Constipation & 11 & $0,2^{*}$ & 9,28 \\
\hline Chills & 10 & 0 & 28 \\
\hline Diarrhea & $\mathrm{I}, 2,12$ & $0^{*}$ & $9,25,26,28,34$ \\
\hline Abdominal pain & & $2^{*}$ & 9 \\
\hline Bone aches & $0,4,10$ & 2,5 & $25,26,34$ \\
\hline Skin rash & 0,1 & 0 & $25,26,34$ \\
\hline Fatigue & $1,6,26$ & 0,5 & $25,28,34$ \\
\hline Cardiovascular & & 8 & 6 \\
\hline Mucositis & & 4 & 6 \\
\hline Anorexia & 12 & 0,1 & 6,28 \\
\hline Sleep disorder & & 1 & 6 \\
\hline Alopecia & & 1 & 6 \\
\hline Anaphylactic reaction & & 1 & 6 \\
\hline Deafness & & 1 & 6 \\
\hline Headache & & I & 6 \\
\hline
\end{tabular}

*Grade 3 and 4 data from the Phase III Decitabine study 9 were combined for the purposes of this review. 
of decitabine. Other rare reported adverse events include pleural effusion and acute fibrinous and organizing pattern of lung injury. ${ }^{43,44}$

Toxicity data that probably most accurately reflect what would be expected across many treatment settings can be taken from randomized Phase II and Phase III studies of decitabine. ${ }^{9,28}$ For instance, in the Phase III study by Kantarjian et al. of the 83 patients treated with decitabine, 69\% experienced severe side effects including neutropenia, thrombocytopenia, febrile neutropenia, leucopenia, pyrexia, hyperbilirubinemia, and pneumonia. ${ }^{9}$ Infrequent side effects included gastrointestinal toxicities $(<5 \%)$. Decitabine dose reductions occurred in only $35 \%$ of patients. In the ADOPT trial, cytopenias were the most common reported grade 3 adverse event at rates of $31 \%$ for neutropenia, $18 \%$ for thrombocytopenia, $14 \%$ for febrile neutropenia, and $12 \%$ for anemia. ${ }^{28}$ Notably, febrile neutropenia occurred most commonly in the first cycle, in particular $10 \%$ of patients. Thirty-two percent of patients had delayed therapy mostly because of myelosuppression at a median of 8 days, and 19\% of administered cycles were associated with a hospitalization. Typical dose reductions of $25 \%-30 \%$ were considered for grade 3 and 4 nonmyelosuppressive toxicities, severe myelosuppressive toxicities, or prolonged bone marrow suppression. ${ }^{25,26,34}$

From the collective experience with decitabine, several general principles emerge. To achieve a maximum therapeutic benefit, delivery of the next scheduled dose of decitabine should not be delayed, unless patients experience disease progression, febrile neutropenia, or profound cytopenias as evident by bone marrow examination ( $<5 \%$ cellular). Results from studies to date demonstrate that the more common adverse effects are transient, nonfatal, and amenable to dose adjustment. Management of adverse effects especially myelosuppression is essential to prevent early discontinuation of decitabine therapy before achieving therapeutic benefit.

\section{Clinical efficacy compared to 5-azacitidine}

In addition to decitabine, 5-azacitidine is the other FDAapproved hypomethylating agent that has become widely used to treat patients with MDS. The clinical benefits of 5 -azacitidine were best demonstrated in the randomized, open-label, landmark Phase III study, which demonstrated better survival than with best conventional care, including chemotherapy. ${ }^{45}$ In this study, 358 patients with higher-risk MDS were randomized to 5-azacitidine or conventional care, which included best supportive care with transfusion products and granulocyte-colony-stimulating factor for neutropenic fever, low-dose cytarabine, or intensive chemotherapy with primary outcome of overall survival. Interestingly, after a median follow up of 21.1 months, patients in the 5-azacitidine arm of the study had a median of 24.5 months overall survival compared to 15 months for patients treated with conventional care (stratified logrank $P=0.0001)$. At 2 years follow-up, $50.8 \%$ of patients on 5 -azacitidine were still alive compared to $26.2 \%$ in the conventional care arm $(P<0.0001)$. Subgroup analysis demonstrated a statistically significant overall survival benefit with 5-azacitidine when compared to best supportive care or cytarabine. Statistical significance was not reached when comparing the study drug to intensive chemotherapy. Secondary endpoints included hematological response, transfusion independence, and hematological improvement. When compared to best supportive care, there was a clear advantage of 5-azacitidine to prolonging transformation to AML by 6 months. Findings of this study suggest longterm treatment with at least 6 , and even up to 9, cycles of 5 -azacitidine may confer the best survival benefit. It remains unknown whether patients with an excess of marrow blasts or unfavorable karyotype would benefit from 5-azacitidine before transplantation.

A common clinical dilemma faced by clinicians is whether one hypomethylating agent provides benefit after a patient fails the other FDA-approved agent. This question has been addressed by a Phase II trial, in which sequential treatment with decitabine in 14 patients with MDS who failed 5-azacitidine was studied. ${ }^{46}$ Early analysis of this Phase II trial looked at the effect of decitabine on the ORR of 14 patients who had MDS and either 5-azacitidine failure, lack of response, or intolerance. Results showed that 3 patients achieved $\mathrm{CR}$ and 1 patient had a marrow $\mathrm{CR}$ with HI, leading to an ORR of $28 \%$.

Clinical trials have not yet compared the treatment advantage of decitabine directly to 5-azacitidine. Although decitabine has shown equal if not better efficacy than azacitidine, there are no prospective data showing increased survival. In the single-center historical study ${ }^{23}$ comparing the outcome of patients with higher-risk MDS when treated with decitabine versus intensive chemotherapy, results suggested a survival advantage for those treated with decitabine (47\% versus $24 \%, P<0.001)$. In contrast, 5 -azacitidine has been shown to have a statistically significant survival advantage (2-year survival, 50.8\% versus 26.2\%, $P<0.0001$ ) when compared to best conventional care in a large, randomized, controlled, multicenter Phase III study. ${ }^{45}$ 
Further, without a randomized comparison of decitabine and 5-azacitidine, selection of the optimal hypomethylating agent for patients diagnosed with MDS remains a challenge for clinicians. Until data from a head-to-head trial are available, two stimulating studies have been published that have generated further interest in the use of hypomethylating agents. In the first study, Gurion et al generated a systematic review and meta-analysis of randomized controlled trials (RCTs) comparing treatment with hypomethylating agents to conventional care in MDS patients. This study suggests 5-azacitidine was superior to decitabine in the treatment of MDS. ${ }^{47}$ The authors analyzed the efficacy of hypomethylating agents versus supportive care for patients with MDS in 4 RCTs that included 952 patients a median age between 67 and 70 years. Results demonstrated a statistically significant overall survival (HR $0.66,95 \%$ CI 0.55 to 0.80 ), and, in particular, an advantage for 5-azacitidine (HR 0.56 , $95 \%$ CI 0.44 to 0.73 ). A survival benefit could not be shown for decitabine (HR 0.88, 95\% CI 0.66 to 1.17). Compared to conventional care, hypomethylating agents slowed progression to AML or death (HR $0.69,95 \%$ CI 0.58 to 0.82 ). Individually, 5-azacitidine clearly had an advantage when compared to conventional care (HR $0.59,95 \%$ CI 0.42 to 0.70) versus decitabine (HR 0.85, 95\% CI 1.41 to 41.17). Surprisingly, treatment-related mortality was associated with hypomethylating agents (RR 7.27, 95\% CI 1.67 to 31.67 ). It is possible that the decitabine data were not as robust given the low median number of courses of treatment offered, since previous studies have demonstrated clinical benefits with at least 6 cycles of therapy.

In a second meta-analysis, Kumar et al compared decitabine and 5-azacitidine in the treatment of MDS using the same RCTs, and their results also uphold the suggested 5 -azacitidine superiority to decitabine. ${ }^{48}$ In the follow-up letter to the editor, Kumar et al further highlighted the differences between the two meta-analyses, including the addition of survival data from the Phase III randomized study by Kantarjian et al and the determination of a clinically nonsignificant difference between 5 -azacitidine and supportive care in the study by Silverman et al. ${ }^{9,49,50}$ Their analysis led to the conclusion that there was no statistically significant overall survival advantage with the use of hypomethylating agents in comparison to supportive care. Similar to the analysis by Gurion et al, pooled data for 5 -azacitidine showed similar overall survival advantage $(0.62,95 \% \mathrm{CI} 0.48$ to $0.78, P=0.030)$. Kumar et al further demonstrated no survival advantage with decitabine ( $0.98,95 \%$ CI 0.81 to $1.18, P=0.815)$. Also, no statistically significant treatment-related mortality was found. Using an adjusted indirect comparison of the two hypomethylating agents, Kumar et al demonstrated a statistically significant survival advantage with 5-azacitidine.

A direct comparison between decitabine and 5-azacitidine is currently underway. Recently, Eisai Corporation of North America initiated a randomized, multicenter, open label study designed as a head-to-head noninferiority trial to test if decitabine is equivalent to 5 -azacitidine in the treatment of patients diagnosed with MDS (Clinicaltrials.gov Identifier NCT01011283). The study plans to enroll 228 adults with Int-1, Int-2, and high-risk MDS who will be randomized to either decitabine or 5 -azacitidine. The primary objective is to compare complete response rates, including bone marrow response rates. Results from this trial are eagerly awaited and may highlight the clinical differences between these two hypomethylating agents.

\section{Synergy with other agents in MDS}

Given the fact that clinical efficacy for single agent decitabine in MDS and AML is at best considered moderate with relatively low CR and PR rates (20\% to $35 \%$ ), there is a need to develop effective combination therapies aimed at improving the response rates, response duration, and eventually survival in these patients. Epigenetic modulation, such as DNA methylation and histone deacetylation can cause reactivation of silenced genes, and in combination, these agents may work better than they can individually. The rationale for this combination emerged from studies demonstrating synergistic effects in the reactivation of epigenetically silenced genes. ${ }^{14,51,52}$ In particular, 5-azacitidine and sodium phenylbutyrate, an HDACi, were used in combination in the Phase I study focused on the treatment of patients diagnosed with MDS and AML, which demonstrated reversal of aberrant epigenetic gene silencing. ${ }^{14}$ Clinical outcomes demonstrated 5 major responders (4 patients with CR and 1 patient with PR) out of 29 evaluable patients in this study. DNA methylation in bone marrow cells was examined by genomic bisulfite sequencing of a hypermethylated $p 15$ promoter. Results from sequential sequencing of DNA in patients who demonstrated PR, CR and HI demonstrated both a significant decrease in $p 15$ methylation following the administration of 5-azacitidine and a pattern of heterogeneous loss of $\mathrm{CpG}$ methylation, suggesting demethylation within the tumor clone. Interestingly, 5-azacitidine alone or in combination with phenylbutyrate led to increased histone acetylation in 17 of 23 patients. Larger studies are needed to evaluate the clinical efficacy of combining DNMT therapy with HDACi in the treatment of MDS. 
Combination therapy of decitabine and valproic acid (VPA, an HDACi) is a well-studied combination. GarciaManero et al evaluated the safety and activity of this combination in a Phase I-II study in 54 elderly patients with MDS and AML. ${ }^{53}$ Based on their preclinical data, ${ }^{54}$ a concomitant dosing schedule was used. The dose of decitabine was $15 \mathrm{mg} / \mathrm{m}^{2}$ intravenously daily for 10 days. In the Phase 1 portion of the study, 3 dose levels of VPA were studied (20, 35 and $50 \mathrm{mg} / \mathrm{kg}$ orally daily for 10 days) and $50 \mathrm{mg} / \mathrm{kg}$ daily was established as maximal tolerated dose (MTD). Of the 32 patients treated at $50 \mathrm{mg} / \mathrm{kg}$ daily VPA dose, 9 developed grade $\geq 3$ nonhematological toxicity, mainly neurotoxicity. Overall, CR was documented in $22 \%$ of the patients. The median time to response was 2 months, and the median response duration was 7.2 months. In the subgroup analysis, $50 \% \mathrm{CR}+\mathrm{CRp}$ (CR with incomplete platelet recovery) was noted in 10 previously untreated patients. Higher VPA levels were associated with higher nonhematological toxicity; however no correlation between VPA levels and response was observed.

In the subgroup analysis restricted to previously untreated patients $(\mathrm{n}=10)$, a $50 \% \mathrm{CR}+\mathrm{CRp}$ (CR with incomplete platelet recovery) was noted, and the responders had significantly higher free VPA levels at day 10 compared to nonresponders $(32.4 \mathrm{mg} / \mathrm{L}$ versus $14.0 \mathrm{mg} / \mathrm{L}, P=0.03)$. Histone $\mathrm{H} 3$ and $\mathrm{H} 4$ acetylation was documented in $35 \%$ of patients at $50 \mathrm{mg} / \mathrm{kg}$ VPA dose. However, no correlation was observed between induction of hypomethylation or histone acetylation and clinical response.

Blum et al conducted a Phase I study using decitabine alone or in combination with VPA in patients with AML. ${ }^{13}$ Twenty-five patients were treated with decitabine dosed at $20 \mathrm{mg} / \mathrm{m}^{2}$ daily IV for 10 days as the optimum biologic dose. VPA was added in escalating doses $(15,20,25 \mathrm{mg} / \mathrm{kg}$ orally daily) for days 5 to 21 , and the MTD for the combination therapy was $20 \mathrm{mg} / \mathrm{kg}$ daily. A CR plus CRi (CR with incomplete count recovery) was noted in 8 patients. There was no difference in the $p 15$ or $E R$ expression levels in patients who received combined therapy versus those who received decitabine at $20 \mathrm{mg} / \mathrm{m}^{2}$ dosing. The authors concluded that the clinical impact of adding VPA to decitabine in this study remained unclear.

Decitabine has also been combined with suberoylanilide hydroxamic acid (SAHA, vorinostat, another HDAC inhibitor). In the preliminary results of a Phase I study reported by Ravandi et al, 5 cohorts of 6 patients each received escalating doses of decitabine $(10,10,15,20$ and $25 \mathrm{mg} / \mathrm{m}^{2}$ intravenously daily for 5 days) followed by vorinostat (100 mg orally 3 times a day for 14 days in the first cohort and $200 \mathrm{mg}$ orally 3 times a day for 14 days in subsequent cohorts). ${ }^{55}$ Two-thirds of the patients had refractory AML. Of the 30 evaluable patients, 1 patient achieved $\mathrm{CR}$, and 4 had significant reduction in the bone marrow blasts. Correlative studies have not been reported for this study.

\section{Conclusions}

The emergence of new treatments for MDS especially hypomethylating agents has generated enthusiasm about a new era of management beyond poorly tolerated cytotoxic therapies and best supportive care. Decitabine has been evaluated primarily in adults diagnosed with higher risk MDS. The potential role of decitabine as a first-line option in patients diagnosed with MDS who are candidates for allogeneic HCT has not been extensively evaluated and presents an opportunity for future research and development. The field also eagerly awaits the results of additional translational studies focusing on decitabine alone or in synergy with other agents to understand its mechanism of action and activity in MDS further. Decitabine has not shown statistically significant evidence of prolonged survival benefits in prospective trials to date, and how its efficacy compares to 5-azacitidine is not yet known. We look forward to the results of the head-to-head randomized trial of decitabine and 5-azacitidine to provide us with further guidance in the management of patients with higher risk MDS.

\section{Abbreviations}

ADOPT, Alternative Dosing for Outpatient Treatment; allogeneic HCT, allogeneic hematopoietic cell transplantation; AML, acute myeloid leukemia; AZA, 5-azacitidine; $\mathrm{BSC}$, best supportive care; CALGB, Cancer and Leukemia Group B; CMML, chronic myelomonocytic leukemia; CR, complete remission; DAC, decitabine; DNA, deoxyribonucleic acid; DNMT, DNA methyltransferase; EORTC, European Organization for Research and Treatment of Cancer; FDA, Food and Drug Administration; HCT, hematopoietic cell transplantation; HDAC, histone deacetylases; HDACi, histone deacetylase inhibitor; HI, hematologic improvement; Int-1, intermediate-1; Int-2, intermediate2; IPSS, International Prognostic Scoring System; mCR, marrow CR; MTD, maximal tolerated dose; mos, months; MPS, myeloproliferative syndrome; NCI, National Cancer Institute; OR, objective response; ORR, overall response rate; PR, partial remission; pts, patients; QOL, quality of life; RBC, red blood cell; RCT, randomized controlled trial; SAHA, suberoylanilide hydroxamic acid; SINE, short 
interspersed element; t-MDS, treatment-related MDS; VPA, valproic acid; WHO, World Health Organization.

\section{Acknowledgments/disclosures}

LAG has received laboratory support from Pharmion, now owned by Celgene.

\section{References}

1. Vardiman JW, Thiele J, Arber DA, et al. The 2008 revision of the World Health Organization (WHO) classification of myeloid neoplasms and acute leukemia: rationale and important changes. Blood. 2009; 114:937-951.

2. Tefferi A, Vardiman JW. Myelodysplastic syndromes. N Engl J Med. 2009;361:1872-1885.

3. Ma X, Does M, Raza A, Mayne ST. Myelodysplastic syndromes: incidence and survival in the United States. Cancer. 2007; 109:1536-1542.

4. Sanz GF, Sanz MA, Greenberg PL. Prognostic factors and scoring systems in myelodysplastic syndromes. Haematologica. 1998;83:358-368.

5. Greenberg P, Cox C, LeBeau MM, et al. International scoring system for evaluating prognosis in myelodysplastic syndromes. Blood. 1997;89:2079-2088.

6. Wijermans PW, Lubbert M, Verhoef G, Klimek V, Bosly A. An epigenetic approach to the treatment of advanced MDS; the experience with the DNA demethylating agent 5-aza-2'-deoxycytidine (decitabine) in 177 patients. Ann Hematol. 2005;84 Suppl 1:9-17.

7. Issa JP, Kantarjian HM. Targeting DNA methylation. Clin Cancer Res. 2009;15:3938-3946.

8. Gore SD, Jones C, Kirkpatrick P. Decitabine. Nat Rev Drug Discov. 2006;5:891-892.

9. Kantarjian H, Issa JP, Rosenfeld CS, et al. Decitabine improves patient outcomes in myelodysplastic syndromes: results of a phase III randomized study. Cancer. 2006;106:1794-1803.

10. Stresemann C, Lyko F. Modes of action of the DNA methyltransferase inhibitors azacytidine and decitabine. Int J Cancer. 2008;123:8-13.

11. Yoo CB, Jeong S, Egger G, et al. Delivery of 5-aza-2'-deoxycytidine to cells using oligodeoxynucleotides. Cancer Res. 2007;67:6400-6408.

12. Kihslinger JE, Godley LA. The use of hypomethylating agents in the treatment of hematologic malignancies. Leuk Lymphoma. 2007;48:1676-1695.

13. Blum W, Klisovic RB, Hackanson B, et al. Phase I study of decitabine alone or in combination with valproic acid in acute myeloid leukemia. J Clin Oncol. 2007;25:3884-3891.

14. Gore SD, Baylin S, Sugar E, et al. Combined DNA methyltransferase and histone deacetylase inhibition in the treatment of myeloid neoplasms. Cancer Res. 2006;66:6361-6369.

15. Figueroa ME, Skrabanek L, Li Y, et al. MDS and secondary AML display unique patterns and abundance of aberrant DNA methylation. Blood. 2009;114:3448-3458.

16. Cheson BD, Bennett JM, Kantarjian H, et al. Report of an international working group to standardize response criteria for myelodysplastic syndromes. Blood. 2000;96:3671-3674.

17. Cheson BD, Greenberg PL, Bennett JM, et al. Clinical application and proposal for modification of the International Working Group (IWG) response criteria in myelodysplasia. Blood. 2006;108:419-425.

18. Rivard GE, Momparler RL, Demers J, et al. Phase I study on 5-aza-2'-deoxycytidine in children with acute leukemia. Leuk Res. 1981;5:453-462.

19. Momparler RL, Rivard GE, Gyger M. Clinical trial on 5-aza2'-deoxycytidine in patients with acute leukemia. Pharmacol Ther. 1985;30:277-286.

20. Willemze R, Archimbaud E, Muus P. Preliminary results with 5-aza2'-deoxycytidine (DAC)-containing chemotherapy in patients with relapsed or refractory acute leukemia. The EORTC Leukemia Cooperative Group. Leukemia. 1993;7 Suppl 1:49-50.
21. Zagonel V, Lo Re G, Marotta G, et al. 5-Aza-2'-deoxycytidine (Decitabine) induces trilineage response in unfavourable myelodysplastic syndromes. Leukemia. 1993;7 Suppl 1:30-35.

22. Wijermans P, Lubbert M, Verhoef G, et al. Low-dose 5-aza2'-deoxycytidine, a DNA hypomethylating agent, for the treatment of high-risk myelodysplastic syndrome: a multicenter phase II study in elderly patients. J Clin Oncol. 2000;18:956-962.

23. Kantarjian HM, O'Brien S, Huang X, et al. Survival advantage with decitabine versus intensive chemotherapy in patients with higher risk myelodysplastic syndrome: comparison with historical experience. Cancer. 2007;109:1133-1137.

24. Wijermans $P$, Suciu S, Baila L, et al. Low dose decitabine versus best supportive care in elderly patients with intermediate or high risk MDS not eligible for intensive chemotherapy: final results of the randomized phase iii study (06011) of the EORTC Leukemia and German MDS Study Groups. 50th American Society of Hematology Annual Meeting. San Francisco, California: Blood. 2008:226.

25. Kantarjian H, Oki Y, Garcia-Manero G, et al. Results of a randomized study of 3 schedules of low-dose decitabine in higher-risk myelodysplastic syndrome and chronic myelomonocytic leukemia. Blood. 2007;109:52-57.

26. Kantarjian HM, O'Brien S, Shan J, et al. Update of the decitabine experience in higher risk myelodysplastic syndrome and analysis of prognostic factors associated with outcome. Cancer. 2007;109:265-273.

27. Ravandi F, Issa JP, Garcia-Manero G, et al. Superior outcome with hypomethylating therapy in patients with acute myeloid leukemia and high-risk myelodysplastic syndrome and chromosome 5 and 7 abnormalities. Cancer. 2009; 115:5746-5751.

28. Steensma DP, Baer MR, Slack JL, et al. Multicenter study of decitabine administered daily for 5 days every 4 weeks to adults with myelodysplastic syndromes: the alternative dosing for outpatient treatment (ADOPT) trial. J Clin Oncol. 2009;27:3842-3848.

29. Ruter B, Wijermans PW, Lubbert M. Superiority of prolonged lowdose azanucleoside administration? Results of 5-aza-2'-deoxycytidine retreatment in high-risk myelodysplasia patients. Cancer. 2006;106:1744-1750.

30. Greenberg PL, Attar E, Battiwalla M, et al. Myelodysplastic syndromes. J Natl Compr Canc Netw. 2008;6:902-926.

31. Germing U, Kundgen A, Gattermann N. Risk assessment in chronic myelomonocytic leukemia (CMML). Leuk Lymphoma. 2004; 45:1311-1318.

32. Wijermans PW, Krulder JW, Huijgens PC, Neve P. Continuous infusion of low-dose 5-Aza-2'-deoxycytidine in elderly patients with high-risk myelodysplastic syndrome. Leukemia. 1997;11:1-5.

33. Wijermans PW, Ruter B, Baer MR, Slack JL, Saba HI, Lubbert M. Efficacy of decitabine in the treatment of patients with chronic myelomonocytic leukemia (CMML). Leuk Res. 2008; 32:587-591.

34. Aribi A, Borthakur G, Ravandi F, et al. Activity of decitabine, a hypomethylating agent, in chronic myelomonocytic leukemia. Cancer. 2007;109:713-717.

35. Scott B, Deeg HJ. Hemopoietic cell transplantation as curative therapy of myelodysplastic syndromes and myeloproliferative disorders. Best Pract Res Clin Haematol. 2006;19:519-533.

36. Kroger N. Epigenetic modulation and other options to improve outcome of stem cell transplantation in MDS. Hematology Am Soc Hematol Educ Program. 2008:60-67.

37. Lubbert M, Hartmut B, Ruter B, Mertelsmann R, Finke J. Nonintensive AML/MDS treatment with low-dose decitabine prior to reduced-intensity conditioning (RIC) and allogeneic blood stem cell transplantation of older patients. 48th American Society of Hematology Annual Meeting. Orlando, FL: Blood. 2006:5257.

38. De Padua S, de Lima M, Kantarjian H, et al. Outcome of allogeneic stem cell transplantation after hypomethylating therapy with 2'-deoxy-5 azacytidine for patients with myelodysplastic syndrome. 49th American Society of Hematology Annual Meeting. Atlanta, GA: Blood. 2007:1468. 
39. Czibere A, Graef T, JL, et al. 5-Azacitidine in combination with donor lymphocyte infusions for the treatment of patients with MDS or AML relapsing after allogeneic stem cell Transplantation. 48th American Society of Hematology Annual Meeting. Orlando, FL: Blood. 2006:5341.

40. de Lima M, Padua L, Giralt S, et al. A dose and schedule finding study of maintenance therapy with low-dose 5-azacitidine (AZA) after allogeneic hematopoietic stem cell transplantation (HSCT) for high-risk AML or MDS. 49th American Society of Hematology Annual Meeting: Blood. 2007:3012.

41. De Padua Silva L, de Lima M, Kantarjian H, et al. Feasibility of alloSCT after hypomethylating therapy with decitabine for myelodysplastic syndrome. Bone Marrow Transplant. 2009;43:839-843.

42. Jain N, Mattiuzzi GN, Cortes J, et al. Benefit of anti-infectious prophylaxis in patients with acute myeloid leukemia or high-risk myelodysplastic syndrome receiving frontline "targeted therapy". 49th American Society of Hematology Annual Meeting. Atlanta, Georgia: Blood. 2007:2858.

43. Chen CC, Gau JP, You JY, et al. Decitabine-induced effusions. Leuk Res. 2009;33:e150-e151.

44. Vasu TS, Cavallazzi R, Hirani A, Marik PE. A 64-year-old male with fever and persistent lung infiltrate. Respir Care. 2009;54:1263-1265.

45. Fenaux P, Mufti GJ, Hellstrom-Lindberg E, et al. Efficacy of azacitidine compared with that of conventional care regimens in the treatment of higher-risk myelodysplastic syndromes: a randomised, open-label, phase III study. Lancet Oncol. 2009;10:223-232.

46. Borthakur G, Ahdab SE, Ravandi F, et al. Activity of decitabine in patients with myelodysplastic syndrome previously treated with azacitidine. Leuk Lymphoma. 2008;49:690-695.

47. Gurion R, Vidal L, Gafter-Gvili A, et al. 5-azacitidine prolongs overall survival in patients with myelodysplastic syndrome - systematic review and meta-analysis. Haematologica. 2009.
48. Kumar A, List AF, Mhaskar R, Djulbegovic B. Efficacy of hypo-methylating agents in the treatment of myelodysplastic syndromes: a systematic review and meta-analysis of randomized controlled trials. 50th American Society of Hematology Annual Meeting. San Francisco, California: Blood. 2008:3632.

49. Kumar A, List AF, Hozo I, Komrokji R, Djulbegovic B. Decitabine versus 5-azacitidine for the treatment of myelodysplastic syndrome: adjusted indirect meta-analysis. Haematologica. 2009.

50. Silverman LR, Demakos EP, Peterson BL, et al. Randomized controlled trial of azacitidine in patients with the myelodysplastic syndrome: a study of the cancer and leukemia group B. J Clin Oncol. 2002;20:2429-2440.

51. Cameron EE, Bachman KE, Myohanen S, Herman JG, Baylin SB. Synergy of demethylation and histone deacetylase inhibition in the reexpression of genes silenced in cancer. Nat Genet. 1999;21:103-107.

52. Suzuki H, Gabrielson E, Chen W, et al. A genomic screen for genes upregulated by demethylation and histone deacetylase inhibition in human colorectal cancer. Nat Genet. 2002;31:141-149.

53. Garcia-Manero G, Kantarjian HM, Sanchez-Gonzalez B, et al. Phase 1/2 study of the combination of 5-aza-2'-deoxycytidine with valproic acid in patients with leukemia. Blood. 2006;108:3271-3279.

54. Yang H, Hoshino K, Sanchez-Gonzalez B, Kantarjian H, GarciaManero G. Antileukemia activity of the combination of 5-aza2'-deoxycytidine with valproic acid. Leuk Res. 2005;29:739-748.

55. Ravandi F, Faderl S, Thomas D, et al. Phase I study of suberoylanilide hydroxamic acid (SAHA) and decitabine in patients with relapsed, refractory or poor prognosis leukemia. 49th American Society of Hematology Annual Meeting. New Orleans, Louisiana: Blood. 2007:897.
OncoTargets and Therapy

\section{Publish your work in this journal}

OncoTargets and Therapy is an international, peer-reviewed, open access journal focusing on the pathological basis of all cancers, potential targets for therapy and treatment protocols employed to improve the management of cancer patients. The journal also focuses on the impact of management programs and new therapeutic agents and protocols on

\section{Dovepress}

patient perspectives such as quality of life, adherence and satisfaction. The manuscript management system is completely online and includes a very quick and fair peer-review system, which is all easy to use. Visit http://www.dovepress.com/testimonials.php to read real quotes from published authors. 